\title{
Discrete symmetries with neutral mesons
}

\author{
José Bernabéu \\ Departamento de Física Teórica, Universidad de Valencia, \\ e IFIC, Joint Centre CSIC-UV, E-46100 Burjassot, Spain
}

\begin{abstract}
Symmetries, and Symmetry Breakings, in the Laws of Physics play a crucial role in Fundamental Science. Parity and Charge Conjugation Violations prompted the consideration of Chiral Fields in the construction of the Standard Model, whereas CP-Violation needed at least three families of Quarks leading to Flavour Physics. In this Lecture I discuss the Conceptual Basis and the present experimental results for a Direct Evidence of Separate Reversal-in-Time T, CP and CPT Genuine Asymmetries in Decaying Particles like Neutral Meson Transitions, using Quantum Entanglement and the Decay as a Filtering Measurement. The eight transitions associated to the Flavour-CP eigenstate decay products of entangled neutral mesons have demonstrated with impressive significance a separate evidence of TRV and CPV in $\mathrm{B}_{\mathrm{d}}$-physics, whereas a CPTV asymmetry shows a $2 \sigma$ effect interpreted as an upper limit. Novel CPTV observables are discussed for $\mathrm{K}$ physics at KLOE-2, including the difference between the semileptonic asymmetries from $\mathrm{K}_{\mathrm{L}}$ and $\mathrm{K}_{\mathrm{S}}$, the ratios of double decay rate Intensities to Flavour-CP eigenstate decay products and the $\omega$-effect. Their observation would lead to a change of paradigm beyond Quantum Field Theory, however there is nothing in Quantum Mechanics forbidding CPTV.
\end{abstract}

\section{The Fundamental Role of Symmetry Breaking}

Symmetries of the Physical Laws, and their Breakings, have played a crucial role in the advance of knowledge. Conservation Laws lead to regularity and order in the description of physical phenomena even when there is no input from dynamics. In the second half of the XXth century, a conceptual revolution appeared when the requirement of local gauge symmetries was understood as originating the interactions themselves with their charges. The emergence of the Standard Model(SM) SU(3) $)_{C} \mathrm{SU}(2)_{\mathrm{L}} \mathrm{X} U(1)_{\mathrm{Y}}$ prompted a spectacular development of particle physics with precise experimental results in agreement with theoretical predictions. The gauge symmetry is exact for massless particles only, which is evidently wrong in Nature. This apparent contradistinction was solved by the BroutEnglert-Higgs mechanism [1] of Spontaneous Breaking of the ElectroWeak Gauge Symmetry $\mathrm{SU}(2)_{\mathrm{L}} \mathrm{x} U(1)_{\mathrm{Y}}$ into $\mathrm{U}(1)_{\mathrm{e} . \mathrm{m}}$. This methodology for explaining the Origin of Mass leaves as a remnant the Higgs Boson, discovered [2] at CERN by the ATLAS and CMS Collaborations.

With respect to the Discrete Symmetries, P, C, CP and T have been observed to be separately broken by weak interactions. Their breakings have had a fundamental imprint in the construction of the SM: 
- Parity Violation, first observed in nuclear beta decay [3] and later, together with the Violation of Charge Conjugation (C), in charged pion decays [4], led to the V-A theory for charged current weak interactions [5] and the role of chirality as the essential ingredient of requiring different transformation properties under the gauge group for left- and righthanded fermionic fields. The chiral fields are the starting components of the theory in the SM. The electroweak unification $[6,7,8]$ culminated with the discovery [9] of neutral current weak interactions by the Gargamelle experiment at CERN.

- CP Violation was unexpectedly discovered in 1964 [10] first in the decay of the long lived neutral Kaon $\mathrm{K}_{\mathrm{L}}$ into two pions, a decay forbidden by the CP Conservation Law. The precision measurements in the Kaon system with secondary Kaon beams and at CPLEAR [11], and specially the observation of CPV in the $B_{d}$ system in the B-Factories $[12,13]$ in 2001 prompted the conviction that the Cabibbo-Kobayashi-Maskawa mechanism of Quark Mixing [14, 15], with at least three families of fermions, is a good description of CPV in the SM. As seen, the origin of the Breaking is here advocated to be in the particle content of the theory. Physical phenomena needing more than one family are included in what we call Flavour Physics.

- A direct evidence of Time Reversal Violation (TRV) is, however, more difficult to be observed. The operator implementing Reversal-in-Time $\mathrm{T}$ in the space of quantum states is Antiunitary [16], in such a way that a true test needs the comparison between the probability for one transition $\mathrm{i} \rightarrow \mathrm{f}$ and that for the $\mathrm{f} \rightarrow \mathrm{i}$ transition. In agreement with the "CPT Theorem" [17] establishing that, under very general assumptions, the description of interactions by means of a Quantum Field Theory (QFT) leads to exact CPT invariance, we should expect TRV for the $K^{0}-\bar{K}^{0}$ and $B_{d}-\bar{B}_{d}$ systems as well. However, these neutral mesons are unstable particles and the decay is irreversible. In words of Wolfenstein [18], "the T-reverse of a decaying particle state is not a physical state", so a NO-GO argument is operating and a direct evidence for TRV appears to be "impossible" for decaying particles. The bypass to this argument came [19, 20] in 1999 from the strategy of not including explicitly the decay products in the TRV asymmetry and using: i) the decay as a filtering measurement of the projected meson state only; ii) Quantum Entanglement of the neutral meson system in order to transfer the information from the decaying meson to its (still alive) orthogonal partner; iii) the asymmetry is searched in the time evolution of this last partner. Under a deep scrutiny of this conceptual basis by many authors, the first observation [21] of TRV in the time evolution of $B_{d}$ 's was announced in 2012 by the BABAR Collaboration, using the methodology that was previously defined in [22]. Its observation triggered $[23,24]$ a revisit to the conditions under which the asymmetry becomes a genuine observable of TRV. For the neutral Kaon system there is a proposal in the DAPHNE-Factory using Entanglement.

- Although P, C, CP and T symmetries have been observed to be separately broken, there is no experimental evidence of CPT Violation. Its observation would imply a change of paradigm to beyond QFT. There is, however, nothing at the level of Quantum Mechanics which forbids CPTV.

In Section 2 we discuss results and proposals for the search of CPTV in Semileptonic Decays of Neutral Kaons. The state of the art for Genuine T, CP, CPT Separate Asymmetries in $B_{d}$ transitions is presented in Section 3. Direct Measurements of TRV in neutral Kaon transitions at KLOE-2 are discussed in Section 4 and the Direct tests of CPT in neutral Kaon transitions at KLOE-2 in Section 5. For entangled Kaons, the possibility of a $\omega$-Effect weakening Entanglement, due to an ill-defined CPT operator, is analysed in Section 6. Conclusions and Outlook are given in Section 7. 


\section{Breaking CPT in SemiLeptonic Decays of Neutral Kaons}

I discuss Tests of the CPT symmetry using the semileptonic decays of neutral Kaons. Assuming the $\Delta S=\Delta \mathrm{Q}$ rule, as implied by the description of flavoured mesons in terms of quarks, the allowed transitions are pictured by the diagrams
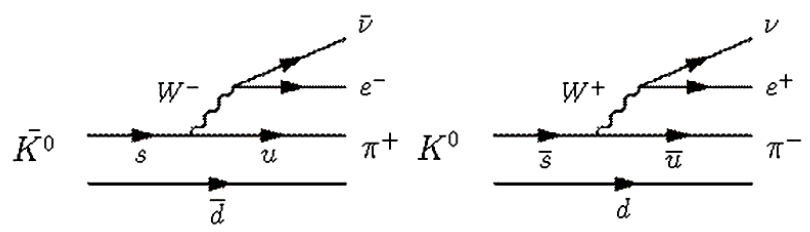

Figure 1- Two decays are allowed according to elementary Quarks ( $\Delta \mathrm{S}=\Delta \mathrm{Q}$ rule)

From the flavour states $K^{0}, \bar{K}^{0}$ the effective Hamiltonian in a Weisskopf-Wigner approach [25] includes a non-diagonal CPV parameter and a diagonal CPTV parameter, so that the states with definite time evolution are

$$
\begin{aligned}
& \left|K_{s}\right\rangle=\frac{1}{\sqrt{2\left(1+\left|\epsilon_{s}\right|^{2}\right)}}\left(\left(1+\epsilon_{s}\right)\left|K^{0}\right\rangle+\left(1-\epsilon_{s}\right)\left|K^{0}\right\rangle\right) \\
& \left|K_{L}\right\rangle=\frac{1}{\sqrt{2\left(1+\left|\epsilon_{L}\right|^{2}\right)}}\left(\left(1+\epsilon_{L}\right)\left|K^{0}\right\rangle-\left(1-\epsilon_{L}\right)\left|K^{0}\right\rangle\right)
\end{aligned}
$$

where

$$
\epsilon_{S / L}=\epsilon_{K} \pm \delta_{K}
$$

where $" \epsilon_{\mathrm{K}}$ " is the parameter describing CPV whereas " $\delta_{\mathrm{K}}$ " is the parameter describing CPTV.

At CPLEAR, the preparation of an initial quantum state of flavour was used. With this tag, the following time-dependent combination of semileptonic decay rates was constructed as an asymmetry

$$
\left\{\begin{array}{c}
A_{\delta}(\tau)=\frac{\bar{R}+(\tau)-\alpha R_{-}(\tau)}{\bar{R}+(\tau)+\alpha R_{-}(\tau)}+\frac{\bar{R}-(\tau)-\alpha R_{+}(\tau)}{\bar{R}-(\tau)+\alpha R_{+}(\tau)} \\
R_{+(-)}(\tau)=R\left(K_{t=0}^{0} \rightarrow\left(e^{+(-)} \pi^{-(+)} v\right)_{t=\tau}\right. \\
\bar{R}_{-(+)}(\tau)=R\left(\bar{K}_{t=0}^{0} \rightarrow\left(e^{-(+)} \pi^{+(-)} v\right)_{t=\tau}\right. \\
\alpha=1+4 \Re \in_{L}
\end{array}\right.
$$

At times outside the interference region, i. e., longer than the short lifetime of $\mathrm{K}_{\mathrm{S}}$, this asymmetry becomes a pure CPT test and determined by the $\mathfrak{R}(\delta)$ parameter as

$$
A_{\delta}\left(\tau \gg \tau_{S}\right)=8 \Re \delta
$$


It is worth noting that $\Re(\delta)$ does not need any absorptive part and it is determined by the difference of diagonal mass terms of $K^{0}$ and $\bar{K}^{0}$. The result of the measurement of the asymmetry (3) by the CPLEAR Collaboration [26] is given in Fig. 2

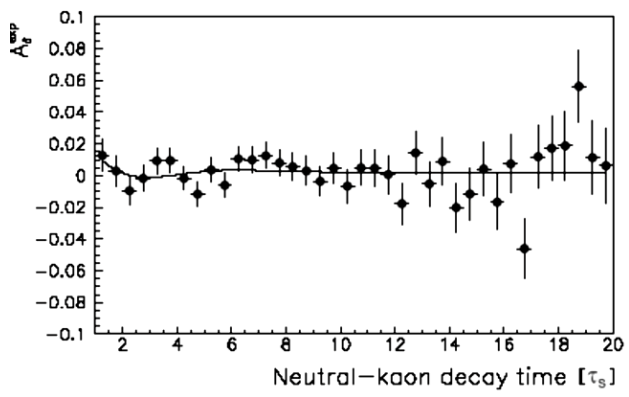

Figure 2-The asymmetry $A_{\delta}(\tau)$ as defined in Eq.3, measured by the CPLEAR Collaboration.

The fit with a constant term produces the result

$$
\Re \delta=(0.30 \pm 0.33 \pm 0.06) \times 10^{-3}
$$

where the first error is statistical and the second systematic.

Its interpretation in terms of the mass matrix elements of the $K^{0}-\bar{K}^{0}$ system gives the upper bound

$$
\left|m_{K^{0}}-m_{\bar{K}^{0}}\right| / m_{K}<10^{-18}
$$

If we take as figure of merit this fractional difference between the masses of a particle and its antiparticle, the result (5) appears to be the best CPT Violation Limit.

At the $\phi$-Factory DAPHNE, the Entanglement between the two neutral Kaon states induced by their antisymmetric exchange allows a separate selection of observable asymmetries from either $\mathrm{K}_{\mathrm{L}}$ or $\mathrm{K}_{\mathrm{S}}$ states. The Charge Asymmetry from these states is given by

$$
A_{S, L}=\frac{\Gamma\left(K_{S, L} \rightarrow \pi^{-} e^{+} v\right)-\Gamma\left(K_{S, L} \rightarrow \pi^{+} e^{-\bar{v}}\right)}{\Gamma\left(K_{S, L} \rightarrow \pi^{-} e^{+} v\right)+\Gamma\left(K_{S, L} \rightarrow \pi^{+} e^{-} \bar{v}\right)}=2\left[\operatorname{Re}\left(\epsilon_{K}\right) \pm \operatorname{Re}\left(\delta_{K}\right)-\operatorname{Re}(y)\right]
$$

where, in addition to the CPV and CPTV parameters in the Hamiltonian matrix, a possible CPTV in the flavour decay amplitudes of Figure 1, parameterised by "y", is included. As seen, the difference between these two Charge Asymmetries $A_{S}-A_{L}$ is able to isolate CPTV effects in the diagonal matrix elements of the Mass Matrix.

The value of $A_{L}$ is well known from hadronic machines, with the best precise result from the KTeV Collaboration [27]

$$
A_{L}=\left(3.332 \pm 0.058_{\text {stat }} \pm 0.047_{\text {syst }}\right) \cdot 10^{-3}
$$

The measurement of $A_{S}$ was undertaken in the first period of the KLOE experiment with the result [28] 


$$
A_{S}=\left(1.5 \pm 9.6_{\text {stat }} \pm 2.9_{\text {syst }}\right) \cdot 10^{-3}
$$

An important improvement of this result is expected at KLOE-2.

\section{Genuine Separate T, CP, CPT Asymmetries in $B_{d}$ transitions}

In Quantum Mechanics, there is an operator $U_{T}$ implementing the T-symmetry acting on the states of the physical system, such that

$$
U_{T} \vec{r} U_{T}^{+}=\vec{r}, U_{T} \vec{p} U_{T}^{+}=-\vec{p}, U_{T} \vec{s} U_{T}^{+}=-\vec{s}
$$

By considering the commutator of the Correspondence Principle,

$$
\left[r_{j}, p_{K}\right]=i \hbar \delta_{j K} I
$$

the operator $U_{T}$, contrary to the case of CP, must be ANTIUNITARY; UNITARY for conserving probabilities, ANTI- for complex conjugation. ANTIUNITARITY introduces many important subtleties, in particular the transition amplitude for $\mathrm{i} \rightarrow \mathrm{f}$ is transformed under $\mathrm{T}$ to

$$
S_{i \rightarrow f} \stackrel{T}{\longrightarrow} S_{U_{T} f \rightarrow U_{T} i}
$$

so that the construction of a Genuine TRV Asymmetry needs the interchange between in and out states.

Something similar can be discussed for the CPT operator, which is ANTIUNITARY too, which needs not only the exchange of in and out states in transitions, but also going from particles to antiparticles.

For unstable particles, the decay products should not be included in the states building the asymmetries for $\mathrm{T}$ or CPT. However, these decay products are what we observe. Therefore, the problem is in the preparation and filtering of the appropriate initial and final meson states from the observed two decay channels. For (Flavour, CP) eigenstate decay products, the Editors of Rev. Mod. Phys. decided to put "what is T-transformation experimentally" in the cover page of the Volume in which the Colloquium article [29] was published. This Figure provides the explanation of what has to be measured in order to build a true genuine TRV asymmetry. With appropriate changes, we reproduce this Figure here, not for TRV but for CPTV. 


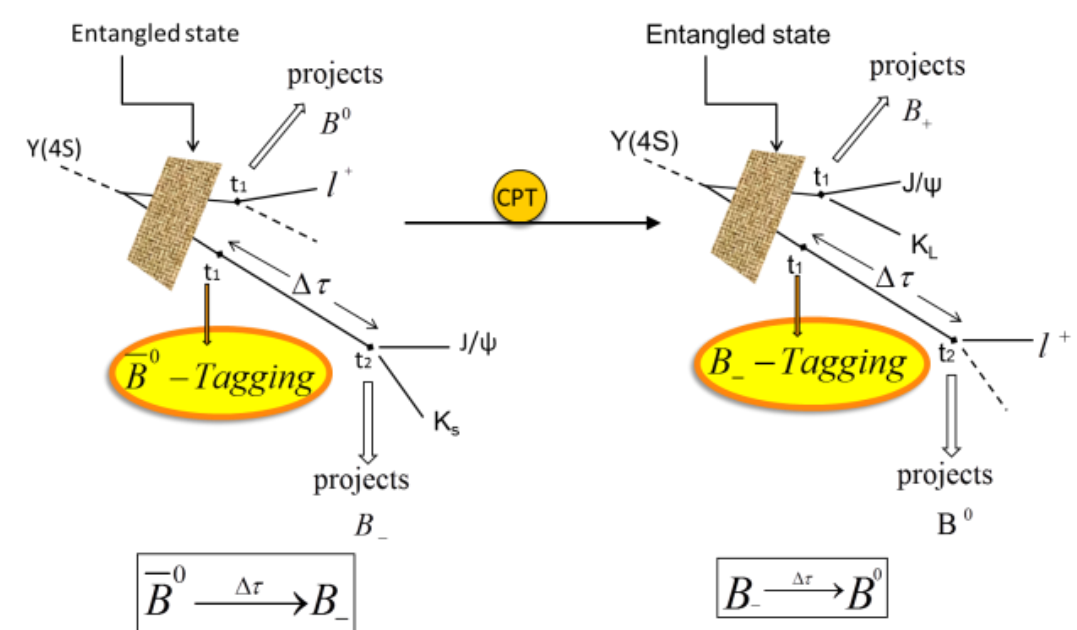

Figure 3 - Experimental CPT transformation

The Figure has illustrated the Reference $\bar{B}^{0} \rightarrow B_{-}$transition, transformed by CPT into the transition $B_{-} \rightarrow B^{0}$. The named neutral meson states $B_{+}, B_{-}$correspond to the filtered states by the $\mathrm{CP}=+$, - eigenstate decay products $\mathrm{J} / \Psi \mathrm{K}_{\mathrm{L}}, \mathrm{J} / \Psi \mathrm{K}_{\mathrm{S}}$.

How many (Flavour, CP) decay channels we have at our disposal experimentally? 2 Flavours x $2 \mathrm{CP} \times 2$ time-ordering $=8$.

These 8 transitions may be grouped in two blocks with extremely important properties. Within each block, the 4 transitions correspond to one Reference and the three different transitions transformed from the Reference by the T, CP and CPT transformations separately. These amazing properties for (Flavour, CP) eigenstate decay channels of the entangled system of neutral mesons are represented in Fig.4, where the time-dependent Intensities of the 8 transitions are organised appropriately in order to make the direct connection of the transitions by the three Discrete Symmetries apparent. The red (left) and blue (right) blocks connected by time exchange are NOT connected by the symmetries.

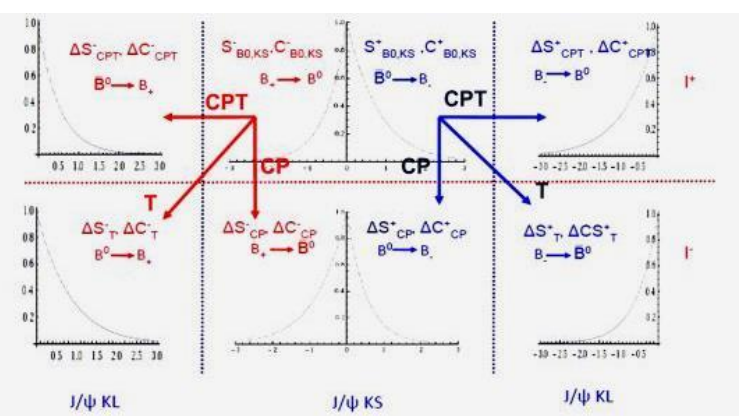

Figure 4 - Reference transition and Separate T, CP, CPT transformed T transitions

Using Quantum Mechanics for the time evolution of the two-state $B^{0}-\bar{B}^{0}$ system and no particular Hamiltonian dynamics, each time dependent Intensity has the following general form

$$
\mathrm{I}(\Delta t) \sim e^{-\Gamma \Delta t}\left\{\mathrm{C}_{C} \cos (\Delta m \Delta t)+S_{c} \sin (\Delta m \Delta t)+\mathrm{C}_{\mathrm{h}} \cosh (\Delta \Gamma \Delta t)+S_{h} \sinh (\Delta \Gamma \Delta t)\right\}
$$


where the coefficients $\mathrm{C}_{\mathrm{c}}, \mathrm{S}_{\mathrm{c}}, \mathrm{C}_{\mathrm{h}}$ and $\mathrm{S}_{\mathrm{h}}$ depend of the selected transition. Without more dynamical theoretical framework, these coefficients are unknown. However, we can construct Asymmetry Parameters $\Delta \mathrm{C}_{\mathrm{c}}, \Delta \mathrm{S}_{\mathrm{c}}$ and $\Delta \mathrm{C}_{\mathrm{h}}$ (in the $\mathrm{B}_{\mathrm{d}}$-system $\Delta \Gamma=0$ ) for each Discrete Symmetry T, CP, CPT. In total, we have 9 Asymmetry Parameters with different information content within each block. Any non-vanishing value of the 3 Asymmetry Parameters for each Symmetry is a bona-fide genuine proof of the Violation of that Symmetry.

In Ref. [30] the authors have worked out the explicit effective Hamiltonian dynamics associated to a Weisskopf-Wigner (WW) quantum description [25] of the meson-antimeson system. In this way the 9 asymmetry parameters can be written in terms of the parameters of the Hamiltonian matrix and the decay amplitudes. Two results are worth mentioning:

i) the Asymmetries are genuine signals of Symmetry Breaking, as they should for any dynamics; ii) the 9 Asymmetry parameters appear with different information content.

Using data from the BABAR analysis [21], in which the Intensities (13) were normalised by dividing by $C_{h}$ with $\Delta \Gamma=0$, the authors of [30] have re-derived the 9 Asymmetry Parameters with some mild additional information. The resulting TRV and CPV asymmetries give the result

$$
\Delta S_{c}^{T}=-0.687 \pm 0.020 ; \Delta S_{c}^{c P}=-0.680 \pm 0.021
$$

These results constitute an impressive separate evidence of TRV and CPV. We emphasize that they are independent results and TRV is established without any assumption on either CPV or CPT invariance.

The analysis performed assuming perfect Entanglement of the neutral meson system in which the two-body state is antisymmetric under the permutation exchange of the two mesons, has also been used to extract the CPTV Asymmetry parameters. The unique antisymmetric state remains unaltered under the time evolution of the entangled system before the first decay, even with Mixing. Under these conditions, given the time ordered (f, g) decays, the reversed time ordering $(\mathrm{g}, \mathrm{f})$ decays satisfy the exchange symmetry conditions

$$
C_{h}(f, g)=C_{h}(g, f) ; C_{c}(f, g)=C_{c}(g, f) ; S_{c}(f, g)=-S_{c}(g, f)
$$

Notice that the exchange symmetry has nothing to do with the discrete symmetries $\mathrm{T}$, $\mathrm{CP}, \mathrm{CPT}$ associated to the dynamics of the system. As a consequence, one has to consider the measurements associated with ( $\mathrm{f}, \mathrm{g})$ and $(\mathrm{g}, \mathrm{f})$ decays as two independent experimental processes measuring the same properties, once Eqs. (15) are imposed. The CPTV Asymmetry is generated in the WW approach by the $\theta$ parameter in the diagonal Hamiltonian matrix elements distinguishing particle and antiparticle properties. The correlated fit to $\operatorname{Re}(\theta)$ and $\operatorname{Im}(\theta)$ is presented [30] in Fig. 5 


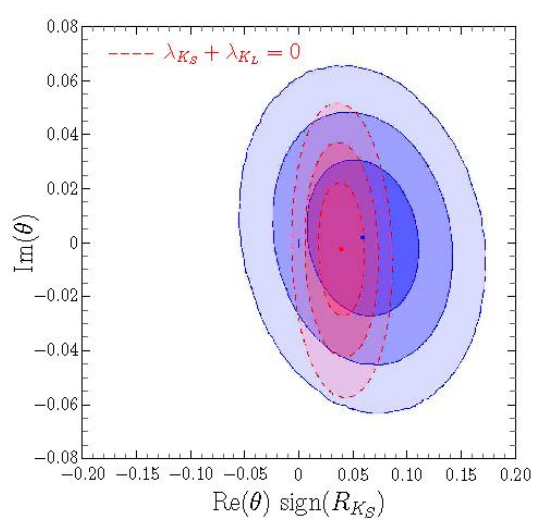

Figure 5 - Correlated fit to the $\operatorname{CPTV} \operatorname{Re}(\theta)$ and $\operatorname{Im}(\theta)$

and gives an intriguing $2 \sigma$ effect for CPTV in $\operatorname{Re}(\theta)$.

Under an ill-defined CPT operator expected in models of quantum gravity, the perfect Entanglement can be weakened [31] by the presence of an $\omega$-effect associated to the "wrong" exchange symmetry of the neutral meson and antimeson. Eqs. (15) are then no longer valid and their violation would then be a signal of this new form of CPT breaking.

\section{Time reversal violation in neutral Kaon transitions}

Similarly to the $B^{0}-\bar{B}^{0}$ system, EPR correlations at a $\phi$ Factory can be exploited to study T-conjugated TRANSITIONS between $K^{0}, \bar{K}^{0}$ states and the orthogonal $\mathrm{K}_{+}, \mathrm{K}$. states filtered by $\mathrm{CP}$ eigenstate decay products

$$
\begin{aligned}
|i\rangle & =\frac{1}{\sqrt{2}}\left[\left|K^{0}(\vec{p})\right\rangle\left|\bar{K}^{0}(-\vec{p})\right\rangle-\left|\bar{K}^{0}(\vec{p})\right\rangle\left|K^{0}(-\vec{p})\right\rangle\right] \\
& =\frac{1}{\sqrt{2}}\left[\left|K_{+}(\vec{p})\right\rangle\left|K_{-}(-\vec{p})\right\rangle-\left|K_{-}(\vec{p})\right\rangle\left|K_{+}(-\vec{p})\right\rangle\right]
\end{aligned}
$$

Using the decays as filtering measurements of the meson states and Entanglement for the preparation of the living partner initial state, we identify the Reference process, for example $K^{0} \rightarrow K_{-}$

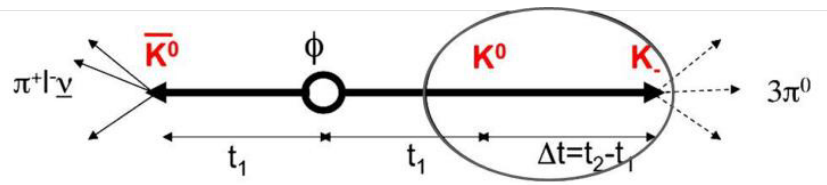

Figure 6 - Neutral Kaon transition $K^{0} \rightarrow K_{-}$filtered by the $1^{-}$decay first, $3 \pi^{0}$ decay later.

For this Reference, the T-conjugated process is $K_{-} \rightarrow K^{0}$, separated from the CP conjugated $\bar{K}^{0} \rightarrow K_{-}$and the CPT-conjugated $K_{-} \rightarrow \bar{K}^{0}$. The T-conjugated process is associated to the time-ordered $2 \pi$ decay first, $1^{+}$decay later 


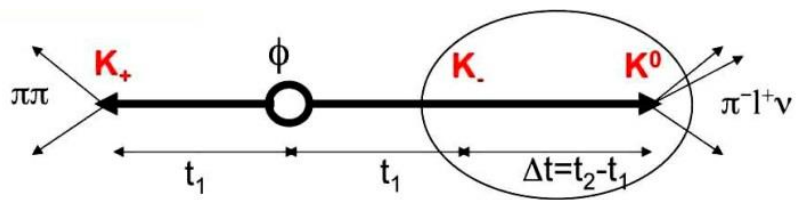

Figure 7 - The Reversal-in-Time Neutral Kaon transition $K_{-} \rightarrow K^{0}$

For a T-symmetry test one has a priori 4 possible comparisons of transitions as given in Table 1

T symmetry test

\begin{tabular}{cc|lc}
\hline \multicolumn{2}{c|}{ Reference } & \multicolumn{2}{c}{$T$-conjugate } \\
Transition & Decay products & \multicolumn{2}{c}{ Transition Decay products } \\
\hline$\overline{\mathrm{K}}^{0} \rightarrow \mathrm{K}_{-}$ & $\left(\ell^{+}, \pi^{0} \pi^{0} \pi^{0}\right)$ & $\mathrm{K}_{-} \rightarrow \overline{\mathrm{K}}^{0}$ & $\left(\pi^{0} \pi^{0} \pi^{0}, \ell^{-}\right)$ \\
$\mathrm{K}_{+} \rightarrow \mathrm{K}^{0}$ & $\left(\pi^{0} \pi^{0} \pi^{0}, \ell^{+}\right)$ & $\mathrm{K}^{0} \rightarrow \mathrm{K}_{+}$ & $\left(\ell^{-}, \pi \pi\right)$ \\
$\overline{\mathrm{K}}^{0} \rightarrow \mathrm{K}_{+}$ & $\left(\ell^{+}, \pi \pi\right)$ & $\mathrm{K}_{+} \rightarrow \overline{\mathrm{K}}^{0}$ & $\left(\pi^{0} \pi^{0} \pi^{0}, \ell^{-}\right)$ \\
$\mathrm{K}_{-} \rightarrow \mathrm{K}^{0}$ & $\left(\pi \pi, \ell^{+}\right)$ & $\mathrm{K}^{0} \rightarrow \mathrm{K}_{-}$ & $\left(\ell^{-}, \pi \pi\right)$ \\
\hline
\end{tabular}

Table 1- Neutral Kaon Transitions to be compared for a T symmetry test

One can then define [33] the following ratios of time dependent probabilities

$$
\begin{aligned}
& R_{1}(\Delta t)=P\left[\mathrm{~K}^{0}(0) \rightarrow \mathrm{K}_{+}(\Delta t)\right] / P\left[\mathrm{~K}_{+}(0) \rightarrow \mathrm{K}^{0}(\Delta t)\right] \\
& R_{2}(\Delta t)=P\left[\mathrm{~K}^{0}(0) \rightarrow \mathrm{K}_{-}(\Delta t)\right] / P\left[\mathrm{~K}_{-}(0) \rightarrow \mathrm{K}^{0}(\Delta t)\right] \\
& R_{3}(\Delta t)=P\left[\overline{\mathrm{K}}^{0}(0) \rightarrow \mathrm{K}_{+}(\Delta t)\right] / P\left[\mathrm{~K}_{+}(0) \rightarrow \overline{\mathrm{K}}^{0}(\Delta t)\right] \\
& R_{4}(\Delta t)=P\left[\overline{\mathrm{K}}^{0}(0) \rightarrow \mathrm{K}_{-}(\Delta t)\right] / P\left[\mathrm{~K}_{-}(0) \rightarrow \overline{\mathrm{K}}^{0}(\Delta t)\right]
\end{aligned}
$$

Any deviation from $\mathrm{R}=1$ constitutes a direct evidence of a Violation of T-symmetry.

These theoretical ratios for meson state transitions can be connected to experimental ratios of Intensities for pairs of decay channels as

$$
\begin{aligned}
R_{1}^{\exp }(\Delta t) & \equiv \frac{I\left(\ell^{-}, \pi \pi ; \Delta t\right)}{I\left(3 \pi^{0}, \ell^{+} ; \Delta t\right)}=R_{1}(\Delta t) \times \frac{C\left(\ell^{-}, \pi \pi\right)}{C\left(3 \pi^{0}, \ell^{+}\right)} \\
R_{2}^{\exp }(\Delta t) & \equiv \frac{I\left(\ell^{-}, 3 \pi^{0} ; \Delta t\right)}{I\left(\pi \pi, \ell^{+} ; \Delta t\right)}=R_{2}(\Delta t) \times \frac{C\left(\ell^{-}, 3 \pi^{0}\right)}{C\left(\pi \pi, \ell^{+}\right)} \\
R_{3}^{\exp }(\Delta t) & \equiv \frac{I\left(\ell^{+}, \pi \pi ; \Delta t\right)}{I\left(3 \pi^{0}, \ell^{-} ; \Delta t\right)}=R_{3}(\Delta t) \times \frac{C\left(\ell^{+}, \pi \pi\right)}{C\left(3 \pi^{0}, \ell^{-}\right)} \\
R_{4}^{\exp }(\Delta t) & \equiv \frac{I\left(\ell^{+}, 3 \pi^{0} ; \Delta t\right)}{I\left(\pi \pi, \ell^{-} ; \Delta t\right)}=R_{4}(\Delta t) \times \frac{C\left(\ell^{+}, 3 \pi^{0}\right)}{C\left(\pi \pi, \ell^{-}\right)}
\end{aligned}
$$

where the co-factors of Eq. (18) are related to the corresponding Branching Ratios. In practice, one has two measurable ratios with the two time-orderings either $\Delta \mathrm{t}>0$ or $\Delta \mathrm{t}<0$, due to the inverse connections

$$
\begin{aligned}
& R_{2}^{\exp }(-\Delta t)=\frac{1}{R_{3}^{\exp }(\Delta t)}=\frac{1}{R_{3}(\Delta t)} \times \frac{C\left(3 \pi^{0}, \ell^{-}\right)}{C\left(\ell^{+}, \pi \pi\right)}, \\
& R_{4}^{\exp }(-\Delta t)=\frac{1}{R_{1}^{\exp }(\Delta t)}=\frac{1}{R_{1}(\Delta t)} \times \frac{C\left(3 \pi^{0}, \ell^{+}\right)}{C\left(\ell^{-}, \pi \pi\right)} .
\end{aligned}
$$


In Figs. 8 we give [32] the Standard Model expectations for the two experimental ratios $R_{2}^{\text {exp }}$ and $R_{4}^{\text {exp }}$, in arbitrary units, as function of $\Delta \mathrm{t}$ for the two time orderings
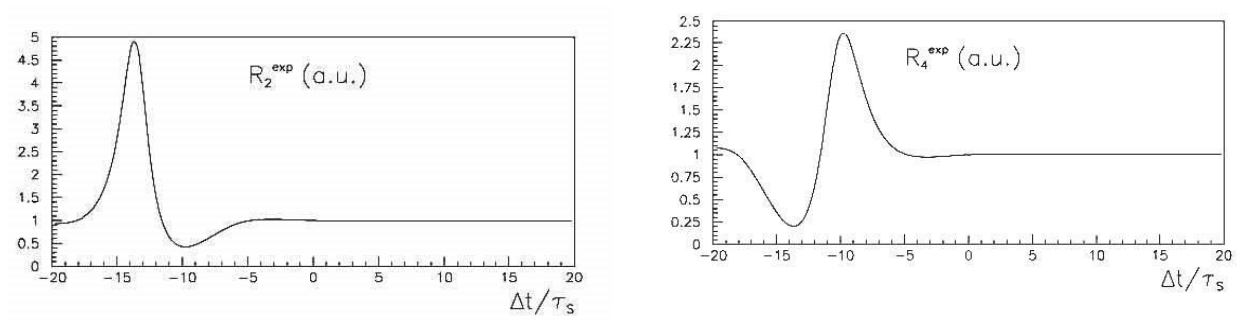

Figure 8 - The experimental ratios $R_{2}^{\text {exp }}$ (left) and $R_{4}^{\text {exp }}$ (right) for TRV expected in the Standard Model

One reaches the conclusion that this T-test could be feasible in KLOE-2 at DAPHNE with a Luminosity of about $10 \mathrm{fb}^{-1}$.

\section{Direct test of CPT in neutral Kaon transitions}

EPR correlations at a $\phi$-Factory can also be exploited to study CPT-conjugated TRANSITIONS involving Flavour $K^{0}, \bar{K}^{0}$ states and the orthogonal $K_{+}, K_{-}$states filtered by $\mathrm{CP}$ eigenstate decay products.

By means of Eq. (16), using the decays as filtering measurements of the meson states and Entanglement for the preparation of the living partner initial state, we identify the Reference process, for example $K^{0} \rightarrow K_{-}$

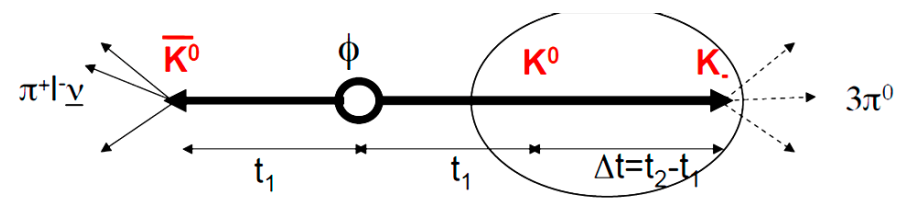

Figure 9- Reference Neutral Kaon Transition $K^{0} \rightarrow K_{-}$associated to the $1^{-}$decay first, $3 \pi^{0}$ decay later.

For this Reference, the CPT-conjugated process is $K_{-} \rightarrow \bar{K}^{0}$, separated out from the CPconjugated $\bar{K}^{0} \rightarrow K_{-}$and the T-conjugated $K_{-} \rightarrow K^{0}$. The CPT-conjugated process is associated to the time-ordered decay channels

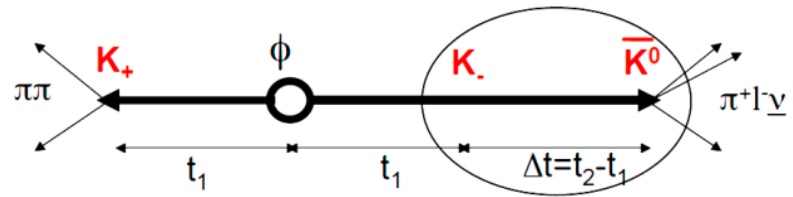

Figure 10- The CPT-transformed $K_{-} \rightarrow \bar{K}^{0}$ is associated to the $2 \pi$ decay first, the $\mathrm{l}^{-}$decay later.

For a CPT-symmetry test one has a priori 4 possible comparisons of transitions as given in Table 2 


\section{CPT symmetry test}

\begin{tabular}{lllll}
\hline Reference & & & \multicolumn{2}{c}{$\mathcal{C} \mathcal{T}$-conjugate } \\
\cline { 1 - 3 } \cline { 5 - 6 } Transition & Decay products & & Transition & Decay products \\
\hline $\mathrm{K}^{0} \rightarrow \mathrm{K}_{+}$ & $\left(\ell^{-}, \pi \pi\right)$ & & $\mathrm{K}_{+} \rightarrow \overline{\mathrm{K}}^{0}$ & $\left(3 \pi^{0}, \ell^{-}\right)$ \\
$\mathrm{K}^{0} \rightarrow \mathrm{K}_{-}$ & $\left(\ell^{-}, 3 \pi^{0}\right)$ & & $\mathrm{K}_{-} \rightarrow \overline{\mathrm{K}}^{0}$ & $\left(\pi \pi, \ell^{-}\right)$ \\
$\overline{\mathrm{K}}^{0} \rightarrow \mathrm{K}_{+}$ & $\left(\ell^{+}, \pi \pi\right)$ & & $\mathrm{K}_{+} \rightarrow \mathrm{K}^{0}$ & $\left(3 \pi^{0}, \ell^{+}\right)$ \\
$\overline{\mathrm{K}}^{0} \rightarrow \mathrm{K}_{-}$ & $\left(\ell^{+}, 3 \pi^{0}\right)$ & & $\mathrm{K}_{-} \rightarrow \mathrm{K}^{0}$ & $\left(\pi \pi, \ell^{+}\right)$ \\
\hline
\end{tabular}

Table 2.- Reference transitions and their CPT-transformed transitions.

One can then define [33] the following ratios of time dependent probabilities

$$
\begin{aligned}
& R_{1, \mathcal{C P} \mathcal{T}}(\Delta t)=P\left[\mathrm{~K}_{+}(0) \rightarrow \overline{\mathrm{K}}^{0}(\Delta t)\right] / P\left[\mathrm{~K}^{0}(0) \rightarrow \mathrm{K}_{+}(\Delta t)\right] \\
& R_{2, \mathcal{C P} \mathcal{T}}(\Delta t)=P\left[\mathrm{~K}^{0}(0) \rightarrow \mathrm{K}_{-}(\Delta t)\right] / P\left[\mathrm{~K}_{-}(0) \rightarrow \overline{\mathrm{K}}^{0}(\Delta t)\right] \\
& R_{3, \mathcal{C P} \mathcal{T}}(\Delta t)=P\left[\mathrm{~K}_{+}(0) \rightarrow \mathrm{K}^{0}(\Delta t)\right] / P\left[\overline{\mathrm{K}}^{0}(0) \rightarrow \mathrm{K}_{+}(\Delta t)\right] \\
& R_{4, \mathcal{C P} \mathcal{T}}(\Delta t)=P\left[\overline{\mathrm{K}}^{0}(0) \rightarrow \mathrm{K}_{-}(\Delta t)\right] / P\left[\mathrm{~K}_{-}(0) \rightarrow \mathrm{K}^{0}(\Delta t)\right]
\end{aligned}
$$

Any deviation from $\mathrm{R}_{\mathrm{i}, \mathrm{CPT}}=1$ constitutes a direct evidence of a Violation of CPTsymmetry.

These theoretical ratios for meson state transitions can be connected to experimental ratios of Intensities for pairs of decay channels as indicated in Table 2.

For visualisation purposes, we plot [33] in Fig. 11 these 4 ratios for CPT-Violation using a " $\delta$ " parameter in the Hamiltonian matrix with values $\operatorname{Re}(\delta)=3.3 .10^{-4}$ and $\operatorname{Im}(\delta)=1.6 .10^{-5}$ (continous line - ) and $\operatorname{Im}(\delta)=0$ (broken line - -).
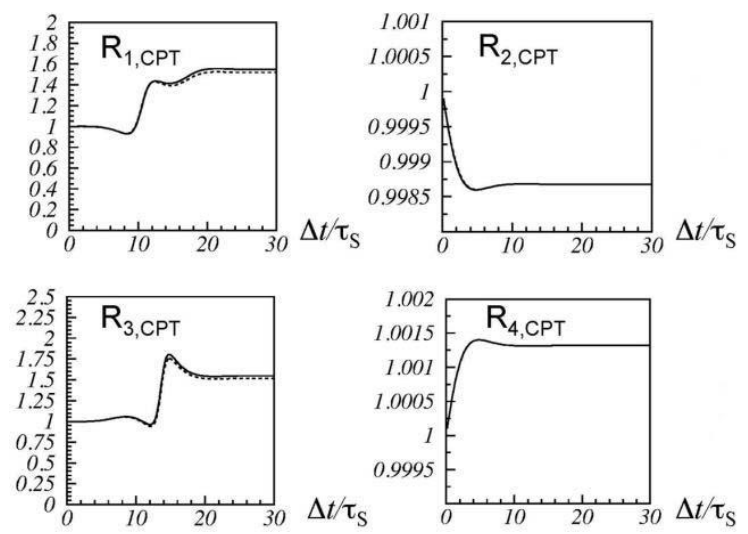

Figure 11.- The ratios of transition probabilities (20) between a Reference and its CPT- transformed for values of the $\delta$ parameter as explained in the text.

For the most interesting $\Delta t$ region larger than the short lifetime of $\mathrm{K}_{\mathrm{S}} \tau_{\mathrm{S}}$, one has

$$
\begin{aligned}
& R_{2, \mathrm{CPT}}\left(\Delta t>>\tau_{\mathrm{S}}\right)=1-4 \operatorname{Re}(\delta) \\
& \mathrm{R}_{4, \mathrm{CPT}}\left(\Delta \mathrm{t}>>\tau_{\mathrm{S}}\right)=1+4 \operatorname{Re}(\delta)
\end{aligned}
$$


One cannot imagine a better clearcut test of CPT symmetry in transitions as the one shown in Eqs. (21). This test is feasible at KLOE-2.

\section{The $\omega$-EFFECT}

In presence of decoherence and CPT breaking induced by quantum gravity effects, the CPT operator is "ill-defined" and the definition of the particle-antiparticle states could be modified. This in turn could induce a weakening of the Entanglement [31] imposed by Bose statistics as an EPR correlation

$$
\begin{aligned}
|i\rangle & \left.\left.\propto\left(\left|K^{0}\right\rangle\left|\bar{K}^{0}\right\rangle-\left|\bar{K}^{0}\right\rangle\left|K^{0}\right\rangle\right)+\infty\left|K^{0}\right\rangle \bar{K}^{0}\right\rangle+\left|\bar{K}^{0}\right\rangle\left|K^{0}\right\rangle\right) \\
& \propto\left(\left|K_{s}\right\rangle\left|K_{L}\right\rangle-\left|K_{L}\right\rangle\left|K_{s}\right\rangle\right)+\omega\left(\left|K_{s}\right\rangle\left|K_{s}\right\rangle-\left|K_{L}\right\rangle\left|K_{L}\right\rangle\right)
\end{aligned}
$$

In some microscopic models of space-time foam [34] with stochastic fluctuations of defect recoils in the propagation of particles, the magnitude of the $\omega$-parameter in Eq. (22) can reach values $\omega \sim 10^{-4}-10^{-5}$.

The maximum sensitivity to $\omega$ is expected for a symmetric decay pair of the entangled state $\mathrm{fl}=\mathrm{f} 2=\pi^{+} \pi^{-}$, which is CP-violating from the dominant antisymmetric state of Eq. (22). As a consequence, one gets a fantastic enhancement of the $\omega$-effect from the "wrong" component in Eq. (22) as $\omega / \varepsilon$, where $\varepsilon$ is the small CPV parameter in K-physics. In Fig. 12 we plot the expected double decay rate time-dependent Intensities for vanishing and nonvanishing values of $\omega$ accessible at KLOE-2.

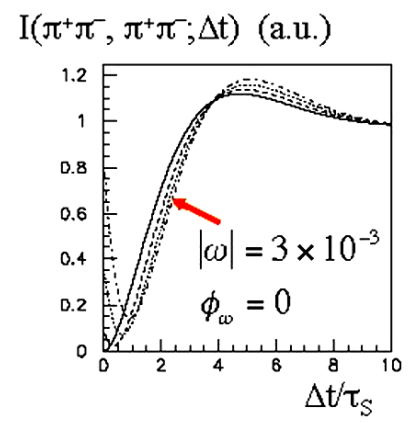

Figure 12.- Time-dependent Intensity for the symmetric pair of decay channels $\left(\pi^{+} \pi^{-}, \pi^{+} \pi^{-}\right)$for vanishing and non-vanishing $\omega$-parameter.

The higher luminosity of present DAPHNE and a good time resolution at KLOE-2 allow to reach an interesting sensitivity to this most fascinating $\omega$-effect.

\section{Conclusions}

I summarize the main Conclusions and Prospects in the studies of the Discrete Symmetries T, CP, CPT with Neutral Mesons:

- The fundamental role that Discrete Symmetry Breaking has played in the understanding of the Laws of Physics emphasizes the importance of looking for Direct Asymmetries of Separate T, CP, CPT in Transitions of Neutral Meson states. In dealing with the Antiunitary $\mathrm{T}$ and $\mathrm{CPT}$, Entanglement is needed.

- The measurement of the semileptonic charge asymmetry from $\mathrm{K}_{\mathrm{S}}$ by KLOE-2 at DAPHNE allows the separation of CPTV in the Mass Matrix of $K^{0}-\bar{K}^{0}$. 
- The Flavour-CP Transitions in Entangled $B^{0}-\bar{B}^{0}$ have demonstrated Genuine Separate Asymmetries for $\mathrm{T}$ and $\mathrm{CP}$ with high statistical significance and, with a $2 \sigma$ tension, compatibility with CPT invariance.

- KLOE-2 is in the best condition to accomplish a complete program of Genuine Separate Asymmetries for T, CP and CPT in Flavour-CP Transitions for $K^{0}-\bar{K}^{0}$. Possible fake effects can be controlled within the same experiment.

- The best way to study the w-effect weakening Entanglement due to an ill-defined CPT operator is the CP-Violating Correlated Decay $\left(\pi^{+}, \pi^{-}, \pi^{+}, \pi^{-}\right)$at KLOE-2. This measurement has a distinctive signature from the dynamical CPTV in the Effective Hamiltonian for $K^{0}-\bar{K}^{0}$.

\section{Acknowledgements}

This research has been supported by MINECO Project FPA 2014-54459-P, Generalitat Valenciana Project GV PROMETEO II 2013-017 and Severo Ochoa Excellence Centre Project SEV 2014-0398.

\section{References}

[1] F. Englert, R. Brout, Phys.Rev.Lett. 13(1964) 321;

P.W. Higgs, Phys.Rev.Lett. 13(1964)508

[2] ATLAS Collaboration, Phys. Lett. B 716(2012)1;

CMS Collaboration, Phys.Lett. B 716(2012)30

[3] T.D.Lee, C.-N. Yang, Phys.Rev. 104(1956)254;

C.S.Wu et al., Phys.Rev. 105(1957)1413

[4] R.L. Garwin et al., Phys. Rev. 105(1957)1415

[5] R.P. Feynman, M. Gell-Mann, Phys.Rev. 109(1958)193

[6] S.L. Glashow, Nucl. Phys. 22(1961)579

[7] S. Weinberg, Phys. Rev. Lett. 19(1967)1264

[8] A. Salam, Conf. Proc. C 680519(1968)367

[9] Gargamelle Neutrino Collaboration, Phys. Lett. B 46 (1973)138

[10] J. H. Christenson et al., Phys. Rev. Lett. 13(1964)138

[11] CPLEAR Collaboration, Phys. Rept. 374(2003)165.

[12] BABAR Collaboration, Phys.Rev.Lett. 87(2001)091801.

[13] Belle Collaboration, Phys.Rev.Lett. 87(2001)091802.

[14] N.Cabibbo, Phys.Rev.Lett. 10(1963)531.

[15] M. Kobayashi, T-Maskawa, Progr.Theor.Phys. 49(1973)652. 
[16] E.P.Wigner, Nachr.Ges.Wiss. Göttingen 32(1932)35

[17] G.Lüders, Annals Phys. 2(1957)1;

W.Pauli et al., "Niels Bohr and the development of Physics", Pergamon NY(1995)

[18] L. Wolfenstein, Phys.Rev.Lett. 83(1999)911.

[19] M.C.Banuls, J.Bernabeu, Phys.Lett.B 464(1999)117

[20] M.C. Banuls, J. Bernabeu, Nucl. Phys. B 590(2000)19

[21] BABAR Collaboration, Phys.Rev.Lett. 109(2012)211801

[22] J. Bernabeu et al., JHEP 1208(2012)064

[23] E. Applebaum et al., Phys.Rev. D 89(2014)076011

[24] J. Bernabeu et al., Phys.Lett. B 728(2014)95

[25] V. Weisskopf, E.P. Wigner, Z. Phys. 63(1930)54;

Z. Phys. 65(1930)18

[26] CPLEAR Collaboration, Phys. Lett. B 444(1998)52

[27] KTeV Collaboration, Phys. Rev. Lett. 88(2002)181601

[28] KLOE Collaboration, Phys. Lett. B 636(2006)173

[29] J. Bernabeu, F. Martinez-Vidal, Rev. Mod. Phys. 87(2015)165

[30] J. Bernabeu et al., JHEP 1606 (2016)100

[31] J. Bernabeu et al., Phys. Rev. Lett. 92(2004)131601

[32] J. Bernabeu et al., Nucl. Phys. B 868(2013)102

[33] J. Bernabeu et al., JHEP 1510(2015)139

[34] J. Bernabeu et al., Phys. Rev. D74(2006)045014 